\title{
Potentiel Physique et Auto-évaluation Corporelle Chez des Jeunes Étudiants à Abidjan
}

\section{Yrobo Yoro Jonathan (Doctorant)}

Département de Paléoanthropologie. Institut des Sciences Anthropologiques de Développement (ISAD), Université Félix Houphouët-Boigny d'Abidjan

Cocody (UFHB). Laboratoire de Biomorphologie.et d'Anthropologie physique rattaché aux Unités de Formation et de Recherche en Sciences

Humaines et Sociales et en OdontoStomatologie de l'UFHB

Kouadio Kouakou Jérôme, (Docteur, Maître-Assistant)

Kouassi Kouakou Firmin (Docteur, Maître-Assistant)

Département de Paléoanthropologie, Institut des Sciences Anthropologiques de Développement (ISAD), Université Félix Houphouët-Boigny d'Abidjan

Cocody (UFHB). Laboratoire de Biomorphologie.et d'Anthropologie physique rattaché aux Unités de Formation et de Recherche en Sciences Humaines et Sociales et en OdontoStomatologie de l'UFHB, Membre de la Société d'Anthropologie de Paris

\section{Beugré Jean-Bertin (Docteur, Maître de Conférences)}

Département d'Orthopédie dento-faciale, Unité de Formation et de

Recherches (UFR) en Odonto-Stomatologie (OS) Département de

Paléoanthropologie, Institut des Sciences Anthropologiques de Développement (ISAD), Université Félix Houphouët-Boigny d'Abidjan Cocody (UFHB). Laboratoire de Biomorphologie.et d'Anthropologie physique rattaché aux Unités de Formation et de Recherche en Sciences Humaines et Sociales et en OS de l'UFHB

Doi: 10.19044/esj.2018.v14n30p113 URL:http://dx.doi.org/10.19044/esj.2018.v14n30p113

\section{Abstract}

The body of the young man seems to be characterized by a specific plasticity. This chrono-individual body modification oftentimes leads the young person to evaluate his own body according to body acquisitions. The relationship between these two variables is far from being sufficiently studied. This paper focuses on examining the self-evaluation of the body according to the physical potential. It covers 181 young people between the ages of 18 and 25, including 84 boys and 97 girls. Body self-judgment of the subjects was measured with a specific scale. In this case, it was measured by the study of Lentillon-Kaestner et al. The physical potential of young people was explored by considering weight, height, Body Mass Index (BMI) and Fat 
Mass Index (FMI) of the latter. The physical parameters were measured using the scales (weight) and the height gauge (stature). As for the body composition indices, they were calculated from the physical parameters previously indicated. The results obtained, after the comparisons of the subjects with each other, show that young people aged 18-21 years of physical threshold potential exhibit statistically high body self-acceptance frequency to that of their subthreshold potential counterparts and suprathreshold physical potential. Similar results were observed in young people between 22 and 25 years of age. In this group, those with a threshold of physical potential were more acceptable compared to their peers of subthreshold physical potential and supraliminal physical potential. Taking into account sex, girls aged 18 to 21 with a threshold physical potential do not differ from their peers with subthreshold physical potential and suprathreshold physical potential in terms of low body self-acceptance. The results are identical for men aged 18 to 21 and 22 to 25 . The meaning of the results does not change when comparing girls with subthreshold physical potential to boys of identical physical potential.The observations made at the latter were repeated when comparing girls to boys of physical threshold potential. In girls aged 22 to 25 , girls with subthreshold physical potential and suprathreshold or threshold were superimposed on their male counterparts of subthreshold physical potential and suprathreshold or threshold. This, however, is in regards to the frequency of body self-acceptance. In this sense, the physical potential of the young would not influence the bodily self-evaluation of the latter when the sex intervenes. On the other hand, considering all young people, their physical density varies based on their body self-evaluation.

Keywords: Physical Ability, Body Standard, Morphotype, Youth, Ivory Coast

\section{Résumé}

Le corps du jeune homme semble se caractériser par une plasticité spécifique. Cette modification corporelle chrono-individuelle amène très souvent le jeune à évaluer lui-même son corps en fonction des acquisitions corporelles. La relation entre ces deux variables est loin d'être suffisamment étudiée. La présente étude se propose d'examiner l'auto-évaluation corporelle selon le potentiel physique. Elle porte sur 181 jeunes âgés de 18 à 25 ans, comprenant 84 garçons et 97 filles. L'auto-jugement corporel des sujets a été mesuré avec une échelle spécifique, en l'occurrence l'échelle de la satisfaction corporelle de Lentillon-Kaestner et al. Le potentiel physique des jeunes a été exploré en considérant le poids, la taille, l'Indice de Masse Corporelle (IMC) et l'Indice de Masse Grasse (IMG) de ces derniers. La mesure des paramètres physiques s'est réalisée par l'utilisation du pèse-personne (poids) et de la toise 
staturale (stature). Les indices relatifs à la composition corporelle ont, quant à eux, été calculés à partir des paramètres physiques précédemment indiqués. Les résultats obtenus, à l'issue des comparaisons des sujets entre eux, montrent que les jeunes âgés de 18-21 ans de potentiel physique liminaire présentent une fréquence d'auto-acceptation corporelle statistiquement élevée à celle de leurs homologues de potentiel physique infraliminaire et ceux de potentiel physique supraliminaire. Des résultats similaires sont observés chez des jeunes dont l'âge varie entre 22 à 25 ans. Dans ce groupe, ceux de niveau de potentiel physique liminaire s'acceptent plus que leurs pairs de potentiel physique infraliminaire et ceux de potentiel physique supraliminaire.Tenant compte du sexe, les jeunes filles âgées de 18 à 21 ans de potentiel physique liminaire ne diffèrent pas de leurs pairs de potentiel physique infraliminaire et de potentiel physique supraliminaire au plan de l'auto-acceptation corporelle bas. Les résultats sont identiques, lorsque l'on considère le sexe masculin, tant chez ceux âgés de 18 à 21 ans et ceux âgés de 22 à 25 ans. Le sens des résultats ne change pas, lorsque l'on compare les filles de potentiel physique infraliminaire aux garçons de potentiel physique identique. Les observations faites chez ces derniers se répètent lorsqu'on compare les filles aux garçons de potentiel physique liminaire. Chez les jeunes de 22 à 25 ans, les filles de potentiel physique infraliminaire; supraliminaire ou liminaire se superposent à leurs homologues garçons de potentiel physique infraliminaire; supraliminaire ou liminaire, concernant la fréquence d'auto-acceptation corporelle. Dans ces sens, le potentiel physique des jeunes n'influencerait donc pas l'auto-évaluation corporelle de ces derniers lorsque le sexe intervient. En revanche, en considérant l'ensemble des jeunes, la densité physique de ces derniers fait varier leur auto-évaluation corporelle.

Mots-clés: Capacité physique, Norme corporelle, Morphotype, Jeunes, Côte d'Ivoire

\section{Introduction}

Le jeune homme semble se caractériser par une structuration physique flexible. Le profil que prend le physique du jeune tend à être généralement examiné par ce dernier, conformément à la morphologie corporelle intrinsèquement programmée. Ce processus de mesure auquel le jeune soumet implicitement son corps renvoie à ce que l'on pourrait appeler 1'autoappréciation corporelle.

Celle-ci se définit comme le sentiment de satisfaction ou non qu'une personne accorde à son propre corps (Neumark-Sztainer et al., 2006). Elle se réfère, selon Jauregui (2011), à la mesure des caractères physiques acquis. L'auto-appréciation corporelle serait donc influencée par le niveau des composantes physiques de l'individu, c'est-à-dire son potentiel physique. Ce 
dernier se réfère à l'ensemble des dimensions physiques dont dispose l'individu (Mercier, 2005). Il renvoie à la qualité des composantes physiques dont un corps peut présenter (Rasmussen et al., 2015). Le potentiel physique du jeune serait donc la densité des acquisitions physiques réalisées par ce dernier.

Ainsi, d'un jeune à l'autre, cette densité corporelle pourrait varier et, par ricochet, être appréciée diversement. Le jugement que le jeune fait de son corps et les différents caractères physiques développés seraient d'autant plus liés que, selon Karastergiou et al. (2016), le corps de l'individu est soumis à un chrono-changement. En d'autres termes, l'auto-appréciation corporelle du jeune dépendrait des acquisitions physiques de celui-ci.

Dans ce sens, Derraik et al. (2016) ont observé des niveaux de perception corporelle différents chez les femmes selon le niveau d'acquisition de la masse grasse adipeuse. Dans une perspective similaire, Bradaric et al. (2015) ont montré que l'acquisition anormale de la structure physique contribue à améliorer une insatisfaction de cette plasticité corporelle. De même, Wang et al. (2010), Pearl et al. (2014) ont mis en évidence la dépréciation corporelle chez les individus présentant des profils physiques déstructurés.

L'on constate que la plupart des études qui tentent d'examiner la relation entre la structuration du corps de l'individu jeune et l'auto-jugement de celui-ci ne s'est pas suffisamment intéressé au potentiel physique comme variable susceptible d'influencer le jugement corporel personnel. L'objectif du présent travail est d'étudier la relation entre le potentiel physique et l'autoévaluation corporelle chez des individus jeunes.

\section{Méthodologie}

\section{Matériel}

Le présent travail s'interroge sur l'auto-appréciation corporelle des jeunes suivant la structuration de leur physique. En effet, les individus de cet âge sont soumis à des changements plus perceptibles de leur corps (Monique, 2000). Cette plasticité corporelle amène le jeune, selon Pica et al. (2012), à une évaluation régulière de celle-ci. La mesure de leur corps par eux-mêmes serait une attitude qui caractériserait les jeunes gens.

De ce point de vue, une telle attention à l'égard de son corps pourrait aussi être observée chez ceux, par exemple, vivant en Côte d'Ivoire. Ces derniers résident, pour la plupart, dans les grandes agglomérations, notamment Abidjan. L'on fait état, dans ce sens, de 348318 jeunes qui vivent dans la ville d'Abidjan (INS, 2014). Ceux-ci se retrouvent en nombre important dans les milieux socioéducatifs. C'est le cas de l'Université Félix Houphouët-Boigny de Cocody. L'on note une proportion importante de jeunes qui fréquentent de façon quotidienne les Unités de Formations de Recherches (UFR) et instituts 
de 1'Université Félix Houphouët-Boigny de Cocody dont 1'Institut des Sciences Anthropologiques de Développement (ISAD) (confère le Service d'inscription de la scolarité centrale de l'Université Félix Houphouët-Boigny, 2015-2016). Cet institut compte 206 jeunes dont l'âge oscille entre 18 et 25 ans. Ces jeunes sont composés de 124 garçons et 102 filles de couche socioéconomique favorisée ou défavorisée, de niveau d'étude variable et ne présentant aucun handicap physique.

En outre, l'on note que ces jeunes sont issus des grands groupes ethnogéographiques de la Côte d'Ivoire, notamment les Akan, les Krou, les Gur, les Mandé. Considérant ces caractéristiques sociodémographiques et sanitaires comme critères d'inclusion ou de non inclusion, 181 jeunes ivoiriens âgés de 18 à 25 ans ont été sélectionnés, dont 84 garçons et 97 filles. C'est l'ensemble de ces jeunes qui constitue l'échantillon sur lequel a porté la présente étude.

\section{Méthodes}

La présente étude de nature transversale et analytique examine l'autoappréciation corporelle des jeunes en rapport avec le potentiel physique de ces derniers. Pour ce faire, les jeunes issus de 1'ISAD-UFHB ont été interrogés. D'abord, l'on a administré à 206, sélectionnés aléatoirement sur 313 de 09 heures à 13 heures 30 minutes, un questionnaire de 20 items portant sur les caractéristiques sociodémographiques des étudiants (le sexe, l’âge, le niveau d'étude, la nationalité, la situation matrimoniale, le statut socioéconomique, l'état corporel). Le dépouillement des items a permis de retenir, en tenant compte de la similarité des caractéristiques, 181 jeunes ivoiriens, dont 84 garçons et 97 filles âgés de 18 à 25 ans, de statut socioéconomique défavorisé.

Le niveau d'auto-acceptation corporelle de ces derniers a été examiné avec une échelle, en l'occurrence l'échelle de la forme corporelle de LentillonKaestner et al. (2010). Cette échelle, comprenant 8 questions, a été soumise de façon collective aux jeunes retenus. Les modalités des réponses aux items de cette échelle sont ordonnées de "jamais" à "toujours", auxquelles sont attribuées des scores. L'obtention d'un score définit une auto-appréciation corporelle positive ou négative (Lentillon-Kaestner et al, op. cit.). Ainsi, si la somme des scores est supérieure à 24, l'individu a une auto-appréciation corporelle positive. Si la somme des scores est inférieure à 24, 1'autoappréciation corporelle est nommée négative (Lentillon-Kaestner et al., op. cit.).

Outre l'exploration du type d'acceptation corporelle, le potentiel physique des jeunes a été examiné à travers la mesure du poids, de la stature, l'Indice de Masse Corporelle (IMC), l'Indice de Masse Grasse (IMG). Les données concernant les auto-appréciations corporelles des jeunes ainsi que le potentiel physique de ces derniers ont été analysées statistiquement au moyen du logiciel SPSS version 23. Les informations relatives aux différents 
paramètres corporels décrivant le potentiel physique des sujets et à l'autoappréciation corporelle ont été respectivement présentées sous forme de moyenne et de fréquence. Par conséquent, le test de Kruskal-wallis Anova à un facteur et celui de khi 2 apparaissent être les tests statistiques appropriés pour vérifier la significativité $(\mathrm{p}<0,05)$ entre les modalités du potentiel physique et celles de l'auto-appréciation corporelle des jeunes.

\section{Résultats}

La relation entre le potentiel physique et l'auto-appréciation corporelle a intéressé la présente étude. Les observations faites, dans ce sens, auprès des jeunes âgés de 18 à 25 ans vivant en Côte d'Ivoire ont abouti à quelques résultats. Les jeunes de 18 à 21 ans de potentiel liminaire, c'est-à-dire d'indice de masse corporelle $\left(21,73 \mathrm{~kg} / \mathrm{m}^{2}\right)$ et grasse $(21,69 \%$ de graisse) équivalent aux normes, présentent un taux d'auto-acceptation corporelle statistiquement supérieur à celui de leurs homologues de potentiel physique infraliminaire (IMC $=17,21 \mathrm{~kg} / \mathrm{m}^{2} ; \quad \mathrm{IMG}=17,01 \%$ de graisse, en dessous des valeurs normative) et de ceux de potentiel physique supraliminaire (IMC $=29,75 \mathrm{~kg} / \mathrm{m}^{2}$; $\mathrm{IMG}=35,71 \%$ de graisse $)(54,4 \%$ contre $36,8 \%$ et $0,0 \%$; avec un $\mathrm{p}$-value $=$ 0,055) (confère le Tableau I).

Tableau I. Comparaison de la fréquence d'auto-évaluation corporelle selon le potentiel physique (IMC, IMG) chez des jeunes âgés de 18 à 21 ans.

\begin{tabular}{cccccccccc}
\hline \multicolumn{4}{c}{ Potentiel Physique } & \multicolumn{4}{c}{ Auto-évaluation corporelle } & Total \\
\hline \multicolumn{2}{c}{ IMC $\left(\mathrm{kg} / \mathrm{m}^{2}\right)$} & \multicolumn{2}{c}{ IMG $(\%)$} & Niveau & \multicolumn{2}{c}{ négative } & positive & $\%$ \\
\hline VO & VN & VO & VN & & n & $\%$ & $\mathrm{n}$ & $\%$ & \\
\hline 17,21 & {$[18,5-24,99]$} & 17,01 & {$[17,5-27,5]$} & PPI & 12 & 63,2 & 7 & 36,8 & 100 \\
\hline 21,73 & {$[18,5-24,99]$} & 21,69 & {$[17,5-27,5]$} & PPL & 31 & 45,6 & 37 & 54,4 & 100 \\
\hline 29,75 & {$[18,5-24,99]$} & 35,71 & {$[17,5-27,5]$} & PPS & 4 & 100 & 0 & 0,0 & 100 \\
\hline & & $\mathrm{p}=0,000 \mathrm{~S}$ & & $\mathrm{p}=0,002 \mathrm{~S}$ & Total & & \multicolumn{2}{c}{$\mathrm{p}$-value (Fischer) $=0,055 \mathrm{NS}$} \\
\hline
\end{tabular}

PPS: Potentiel Physique Supraliminaire; PPI: Potentiel Physique Infraliminaire; PPL: Potentiel Physique Liminaire; n: Effectif; VO: Valeur Observée; VN: Valeur Normative, IMC: Indice de Masse Corporelle; IMG: Indice de Masse Grasse; \%: pourcentage; NS: Non Significative; S: Significative; kg: kilogramme; m: mètre.

Une différence au niveau de l'auto-satisfaction corporelle entre les trois groupes est également observée, lorsque l'on compare les jeunes de 22 à 25 ans entre eux. Les potentiels physiques liminaires $(\mathrm{IMC}=21,64 \mathrm{~kg} / \mathrm{m} 2$, $\mathrm{IMG}=19,5 \%$ de graisse) s'acceptent plus que les potentiels physiques infraliminaires $\left(\mathrm{IMC}=17,37 \mathrm{~kg} / \mathrm{m}^{2}, \mathrm{IMG}=15,15 \%\right.$ de graisse) et les potentiels physiques supraliminaires (IMC $=30,28 \mathrm{~kg} / \mathrm{m}^{2}, \quad \mathrm{IMG}=33,04 \%$ de graisse) $(58,2 \%$ contre $50,2 \%$, et $14,3 \%$; p-value $=0,010)$ (confère le Tableau II). 
Tableau II. Comparaison de la fréquence d'auto-évaluation corporelle en fonction du potentiel physique (IMC, IMG) chez des jeunes âgés de 22 à 25 ans

\begin{tabular}{|c|c|c|c|c|c|c|c|c|c|}
\hline \multicolumn{5}{|c|}{ Potentiel Physique } & \multicolumn{4}{|c|}{ Auto-évaluation corporelle } & \multirow{3}{*}{$\begin{array}{c}\text { Total } \\
\% \\
\end{array}$} \\
\hline \multicolumn{2}{|c|}{$\operatorname{IMC}\left(\mathrm{kg} / \mathrm{m}^{2}\right)$} & \multicolumn{2}{|c|}{ IMG (\%) } & \multirow[t]{2}{*}{ Niveau } & \multicolumn{2}{|c|}{ négative } & \multicolumn{2}{|c|}{ positive } & \\
\hline $\mathrm{VO}$ & $\mathrm{VN}$ & $\mathrm{VO}$ & $\mathrm{VN}$ & & $\mathrm{n}$ & $\%$ & $\mathrm{n}$ & $\%$ & \\
\hline 17,37 & {$[18,5-24,99]$} & 15,15 & {$[17,5-27,5]$} & PPI & 7 & 49,8 & 9 & 50,2 & 100 \\
\hline 21,64 & {$[18,5-24,99]$} & 19,50 & {$[17,5-27,5]$} & PPL & 28 & 41,8 & 39 & 58,2 & 100 \\
\hline \multirow{2}{*}{\multicolumn{2}{|c|}{$[18,5-24,99]$}} & 33,04 & {$[17,5-27,5]$} & PPS & 6 & 85,7 & 1 & 14,3 & 100 \\
\hline & & & & Total & 37 & 41,1 & 53 & 58,9 & 100 \\
\hline & $0,000 \mathrm{~S}$ & &, $000 \mathrm{~S}$ & & & value ( & er) $=$ & $10 \mathrm{~S}$ & \\
\hline
\end{tabular}

PPS: Potentiel Physique Supraliminaire; PPI: Potentiel Physique Infraliminaire; PPL: Potentiel Physique Liminaire; n: Effectif; VO: Valeur Observée; VN: Valeur Normative, IMC: Indice de Masse Corporelle; IMG: Indice de Masse Grasse; \%: pourcentage; NS: Non Significative; S: Significative; kg: kilogramme; m: mètre.

L'auto-appréciation corporelle qui varie de façon générale chez les jeunes, quel que soit leur potentiel physique, est observée en tenant compte du sexe (filles et garçons). En effet, comparées à leurs pairs de potentiel physique infraliminaire (IMC $=17,22 \mathrm{~kg} / \mathrm{m}^{2}, \mathrm{IMG}=20,54 \%$ de graisse) et supraliminaire (IMC=29,75 kg/m², IMG=35,71\% de graisse), les filles âgées de 18 à 21 ans de potentiel physique liminaire (IMC $=21,84 \mathrm{~kg} / \mathrm{m}^{2}, \mathrm{IMG}=26,40 \%$ de graisse) acceptent leur structure physique, à un pourcentage analogue à celui de leur pairs de potentiel physique infraliminaire et supraliminaire $(53,8 \%$ contre $33,3 \%$ et $0,0 \%$; $p$-value $=0,087$ ) (confère le Tableau III).

Tableau III. Comparaison de la fréquence d'auto-évaluation corporelle des filles ou des garçons de 18 à 21 ans entre elles ou eux selon le potentiel physique

\begin{tabular}{|c|c|c|c|c|c|c|c|c|c|c|}
\hline \multicolumn{4}{|c|}{ Potentiel physique } & & \multirow[t]{3}{*}{ Sexe } & \multicolumn{4}{|c|}{ Auto-évaluation corporelle } & Total \% \\
\hline \multicolumn{2}{|c|}{ IMC $\left(\mathrm{kg} / \mathrm{m}^{2}\right)$} & \multicolumn{2}{|c|}{ IMG $(\%)$} & \multirow[t]{2}{*}{ Niveau } & & \multicolumn{2}{|c|}{ Négative } & \multicolumn{2}{|c|}{ Positive } & \\
\hline VO & $\mathrm{VN}$ & VO & VN & & & $\mathrm{n}$ & $\%$ & $\mathrm{n}$ & $\%$ & \\
\hline 17,22 & {$[18,5-24,99]$} & 20,54 & [25-30] & PPI & $\mathrm{F}$ & 12 & 66,7 & 6 & 33,3 & 100 \\
\hline 21,84 & {$[18,5-24,99]$} & 26,40 & [25-30] & PPL & $\mathrm{F}$ & 18 & 46,2 & 21 & 53,8 & 100 \\
\hline \multirow[t]{2}{*}{29,75} & {$[18,5-24,99]$} & 35,71 & [25-30] & PPS & F & 4 & 100 & 0 & 0,0 & 100 \\
\hline & $0,000 \mathrm{~S}$ & \multicolumn{2}{|c|}{$\mathrm{p}=0,000 \mathrm{~S}$} & & & \multicolumn{4}{|c|}{ Fischer $(\mathrm{p}$-value $)=0,087 \mathrm{NS}$} & \\
\hline 17 & {$[18,5-24,99]$} & 10,55 & [15-20] & PPI & G & 0 & 0,0 & 1 & 100 & 100 \\
\hline 21,58 & {$[18,5-24,99]$} & 15,34 & [15-20] & PPL & G & 13 & 44,8 & 16 & 55,2 & 100 \\
\hline 0 & {$[18,5-24,99]$} & 0 & [15-20] & PPS & $\mathrm{G}$ & 0 & 0,0 & 0 & 0,0 & 0,0 \\
\hline \multicolumn{2}{|c|}{$\mathrm{p}=0,005 \mathrm{~S}$} & \multicolumn{2}{|c|}{$p=0,003 S$} & & & \multicolumn{4}{|c|}{ Fischer $(p-$ value $)=0,567 \mathrm{NS}$} & \\
\hline
\end{tabular}

PPS: Potentiel Physique Supraliminaire; PPI: Potentiel Physique Infraliminaire; PPL: Potentiel Physique Liminaire; n: Effectif; VO: Valeur Observée; VN: Valeur Normative, IMC: Indice de Masse Corporelle; IMG: Indice de Masse Grasse; \%: pourcentage; NS: Non Significative; S: Significative; kg: kilogramme; m: mètre; F: Fille; G: Garçon.

L'effet non significatif du potentiel physique sur l'auto-appréciation corporelle semble être constant, puisque les résultats observés chez les garçons 
ne s'écartent pas de ceux de leurs homologues filles. Les garçons présentant un potentiel physique liminaire ( $\mathrm{IMC}=21,58 \mathrm{~kg} / \mathrm{m}^{2}, \mathrm{IMG}=15,34 \%$ de graisse) acceptent également leur corps que leurs homologues de potentiel physique infraliminaire $\left(\mathrm{IMC}=17 \mathrm{~kg} / \mathrm{m}^{2}, \mathrm{IMG}=10,55 \%\right.$ de graisse) $(55,2 \%$ contre 100\%; p-value=0,567) (confère le Tableau III). Ces résultats pourraient se généraliser, lorsqu'on passe d'une tranche d'âge à une autre.

Ainsi, les filles âgées de 22 à 25 ans de potentiel physique liminaire $\left(\mathrm{IMC}=22,04 \mathrm{~kg} / \mathrm{m}^{2}, \mathrm{IMG}=27,07 \%\right.$ de graisse) présentent une fréquence d'acceptation corporelle comparable à celle de leurs pairs filles de niveau de potentiel physique infraliminaire $\left(\mathrm{IMC}=17,57 \mathrm{~kg} / \mathrm{m}^{2}, \quad \mathrm{IMG}=21,17 \%\right.$ de graisse) et de niveau de potentiel physique supraliminaire (IMC $=30,83 \mathrm{~kg} / \mathrm{m}^{2}$, $\mathrm{IMG}=34,82 \%$ de graisse $)(47,8 \%$ contre $100 \%$ et $16,7 \%$; p-value $=0,066)$ (confère le Tableau IV). Des résultats statistiques similaires sont observés chez les garçons du même âge. En d'autres termes, les garçons de potentiel physique supraliminaire rejettent leur corps à un pourcentage invariable à celui de leurs pairs de potentiel physique liminaire, c'est-à-dire dont l'IMC=21,43 $\mathrm{kg} / \mathrm{m}^{2}$; l' $\mathrm{IMG}=15,54 \%$ de graisse et ceux de potentiel physique infraliminaire (l' IMC $=17,22 \mathrm{~kg} / \mathrm{m}^{2}, l^{\prime} \mathrm{IMG}=10,40 \%$ de graisse) $(100 \%$ contre $36,4 \%$ et $33,3 \%$; p-value $=0,542$ ) (confère le Tableau IV). Les résultats relatifs à l'effet $\mathrm{du}$ dimorphisme sexuel sur la relation entre le potentiel physique et l'autoappréciation corporelle apportent des informations nouvelles.

Tableau IV. Comparaison de la fréquence d'auto-évaluation corporelle des filles ou des garçons de 22 à 25 ans entre elles ou eux en fonction du potentiel physique

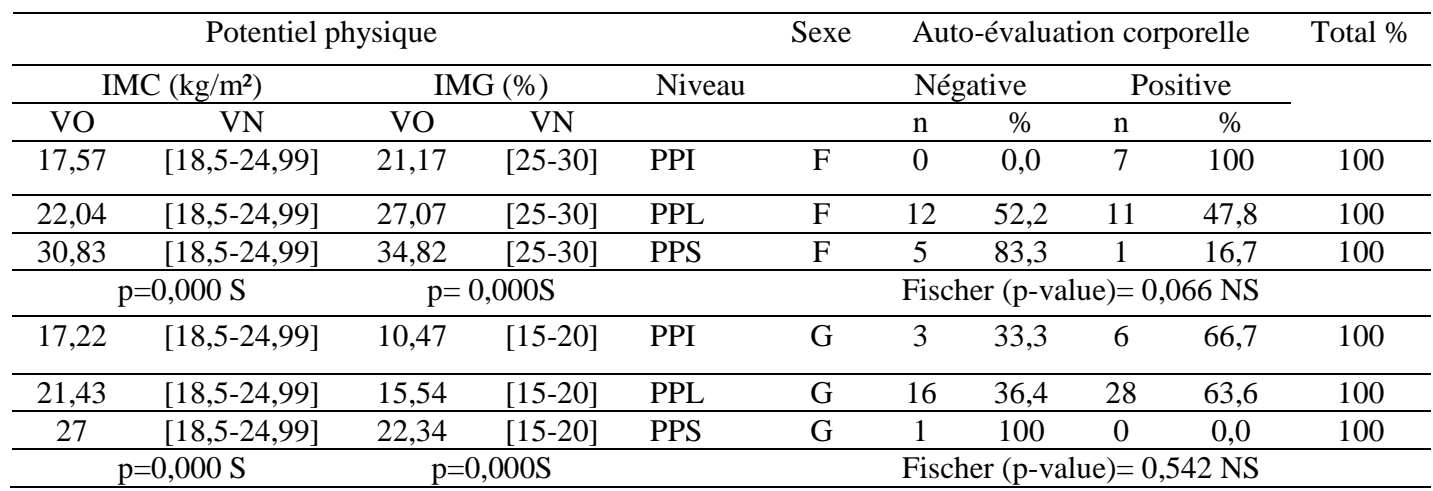

PPS: Potentiel Physique Supraliminaire; PPI: Potentiel Physique Infraliminaire; PPL: Potentiel Physique Liminaire; n: Effectif; VO: Valeur Observée; VN: Valeur Normative, IMC: Indice de Masse Corporelle; IMG: Indice de Masse Grasse; \%: pourcentage; NS: Non Significative; S: Significative; kg: kilogramme; m: mètre; F: Fille; G: Garçon.

Des résultats de comparaisons entre les garçons et les filles de potentiel physique identique ont été relevés. Dans ce sens, l'on note que, à potentiel physique infraliminaire, les garçons âgés de 18 à 21 ans $\left(\mathrm{IMC}=17,00 \mathrm{~kg} / \mathrm{m}^{2}\right.$, $\mathrm{IMG}=10,55 \%$ de graisse) ont tendance à accepter leur profil physique à une fréquence statistiquement similaire à celle de leurs homologues filles 
(IMC $=17,22 \mathrm{~kg} / \mathrm{m}^{2}, \quad \mathrm{IMG}=20,54 \%$ de graisse $)(100 \%$ contre $33,3 \% ; \mathrm{p}-$ value $=0,179$ ) (confère le Tableau V). En considérant le potentiel physique supraliminaire identique, les filles âgés de 18 à 21 ans $\left(\mathrm{IMC}=29,75 \mathrm{~kg} / \mathrm{m}^{2}\right.$, $\mathrm{IMG}=35,71 \%$ de graisse) sont présentes dans 1'auto-appréciation corporelle basse $(100 \%)$. Par contre, il existe aucuns garçons dans le potentiel physique supraliminaire (confère le Tableau V). A potentiel physique liminaire, les filles de cette tranche d'âge ( $\mathrm{IMC}=21,78 \mathrm{~kg} / \mathrm{m}^{2}, \mathrm{IMG}=26,44 \%$ de graisse) présentent un taux d'auto-appréciation corporelle équivalent à celui de leurs homologues garçons ( $\mathrm{IMC}=21,58 \mathrm{~kg} / \mathrm{m}^{2}, \mathrm{IMG}=15,34 \%$ de graisse) $(53,8 \%$ contre 55,2\%; p-value=0,914) (confère le Tableau V). Des résultats identiques semblent se répétés lorsqu'on passe d'une tranche d'âge à une autre.

$\underline{\text { Tableau V. }}$. Comparaison de la fréquence d'auto-évaluation corporelle des filles de 18 à 21 ans à celle de leurs pairs garçons de potentiel physique similaire.

\begin{tabular}{|c|c|c|c|c|c|c|c|c|c|c|}
\hline \multicolumn{5}{|c|}{ Potentiel physique } & \multirow[t]{3}{*}{ Sexe } & \multicolumn{4}{|c|}{ Auto-évaluation corporelle } & \multirow[t]{3}{*}{ Total $(\%)$} \\
\hline \multicolumn{2}{|c|}{$\operatorname{IMC~}\left(\mathrm{kg} / \mathrm{m}^{2}\right)$} & \multicolumn{2}{|c|}{ IMG $(\%)$} & \multirow[t]{2}{*}{ Niveau } & & \multicolumn{2}{|c|}{ Négatif } & \multicolumn{2}{|c|}{ Positive } & \\
\hline VO & $\mathrm{VN}$ & $\mathrm{VO}$ & $\mathrm{VN}$ & & & $\mathrm{n}$ & $\%$ & $\mathrm{n}$ & $\%$ & \\
\hline 17,22 & {$[18,5-24,99]$} & 20,54 & [25-30] & & $\mathrm{F}$ & 12 & 66,7 & 6 & 33,3 & 100 \\
\hline 17,00 & {$[18,5-24,99]$} & 10,55 & [15-20] & PPI & $\mathrm{G}$ & 0 & 0,0 & 1 & 100 & 100 \\
\hline \multicolumn{2}{|c|}{$\mathrm{p}$-value $=0,695 \mathrm{NS}$} & \multicolumn{2}{|c|}{ p-value $=0,098 \mathrm{NS}$} & & & \multicolumn{5}{|c|}{ Fisher $(p$-value $)=0,179 \mathrm{NS}$} \\
\hline 21,78 & {$[18,5-24,99]$} & 26,44 & {$[25-30]$} & \multirow[b]{2}{*}{ PPL } & $\mathrm{F}$ & 18 & 46,2 & 21 & 53,8 & 100 \\
\hline 21,58 & {$[18,5-24,99]$} & 15,34 & [15-20] & & G & 13 & 44,8 & 16 & 55,2 & 100 \\
\hline \multicolumn{2}{|c|}{$\mathrm{p}$-value $=0,861 \mathrm{NS}$} & \multicolumn{2}{|c|}{ p-value $=0,000 \mathrm{~S}$} & & \multicolumn{6}{|c|}{ Fisher $(p$-value $)=0,914 \mathrm{NS}$} \\
\hline 29,75 & {$[18,5-24,99]$} & 35,71 & [25-30] & & $\mathrm{F}$ & 2 & 100 & 0 & 0,0 & 100 \\
\hline 0,0 & {$[18,5-24,99]$} & 0,0 & {$[15-20]$} & PPS & G & 0 & 0,0 & 0 & 0,0 & 0 \\
\hline
\end{tabular}

p-value $=1,000 \mathrm{NS}$-value $=1,000 \mathrm{NS}$

Fisher (p-value) $=0,046 \mathrm{~S}$

PPS: Potentiel Physique Supraliminaire; PPI: Potentiel Physique Infraliminaire; PPL: Potentiel Physique Liminaire; n: Effectif; VO: Valeur Observée; VN: Valeur Normative, IMC: Indice de Masse Corporelle; IMG: Indice de Masse Grasse; \%: pourcentage; NS: Non Significative; S: Significative; kg: kilogramme; m: mètre; F: Fille; G: Garçon.

De ce fait, à potentiel physique infraliminaire, les garçons âgés de 22 à 25 ans $\left(\mathrm{IMC}=17,22 \mathrm{~kg} / \mathrm{m}^{2}, \mathrm{IMG}=10,47 \%\right.$ de graisse) acceptent leur corps statistiquement comparable à celui de leurs homologues filles (d'IMC $=17,57 \mathrm{~kg} / \mathrm{m}^{2}, \quad I M G=21,17 \%$ de graisse) $(66,7 \%$ contre $100 \%$; pvalue $=0,090)$ (confère le Tableau VI). 
Tableau VI. Comparaison de la fréquence d'auto-évaluation corporelle des filles de 22 à 25 ans à celle de leurs pairs garçons de potentiel physique identique.

\begin{tabular}{|c|c|c|c|c|c|c|c|c|c|}
\hline \multicolumn{4}{|c|}{ Potentiel physique } & \multirow[t]{3}{*}{ Sexe } & \multicolumn{4}{|c|}{ Auto-évaluation corporelle } & \multirow[t]{3}{*}{ Total $(\%)$} \\
\hline $\operatorname{IMC}\left(\mathrm{kg} / \mathrm{m}^{2}\right)$ & \multicolumn{2}{|c|}{ IMG $(\%)$} & \multirow[t]{2}{*}{ Niveau } & & \multicolumn{2}{|c|}{ Négatif } & \multicolumn{2}{|c|}{ Positive } & \\
\hline $\mathrm{VO} \quad \mathrm{VN}$ & $\mathrm{VO}$ & $\mathrm{VN}$ & & & $\mathrm{n}$ & $\%$ & $\mathrm{n}$ & $\%$ & \\
\hline$[18,5-24,99]$ & 21,17 & [25-30] & & $\mathrm{F}$ & 0 & 0,00 & 7 & 100 & 100 \\
\hline [18,5-24,99] & 10,47 & [15-20] & PPI & $\bar{G}$ & 3 & 33,3 & 6 & 66,7 & 100 \\
\hline $\mathrm{p}$-value $=0,166 \mathrm{NS}$ & \multicolumn{2}{|c|}{$\mathrm{p}$-value $=0,001 \mathrm{~S}$} & & & \multicolumn{5}{|c|}{ Fisher $(\mathrm{p}$-value $)=0,090 \mathrm{NS}$} \\
\hline$[18,5-24,99]$ & 27,07 & [25-30] & & $\mathrm{F}$ & 12 & 52,2 & 11 & 47,8 & 100 \\
\hline$[18,5-24,99]$ & 15,54 & [15-20] & PPL & $\mathrm{G}$ & 16 & 36,4 & 28 & 63,6 & 100 \\
\hline P-value $=0,030 \mathrm{~S}$ & \multicolumn{2}{|c|}{ P-value $=0,000 \mathrm{~S}$} & & & \multicolumn{4}{|c|}{ Fisher $(\mathrm{p}$-value $)=0,213 \mathrm{NS}$} & \\
\hline$[18,5-24,99]$ & 34,82 & [25-30] & & $\mathrm{F}$ & 5 & 83,3 & 1 & 16,7 & 100 \\
\hline $27,0 \quad[18,5-24,99]$ & 22,34 & {$[15-20]$} & PPS & $\mathrm{G}$ & 1 & 100 & 0 & 0,0 & 100 \\
\hline $\mathrm{p}$-value $=0,799 \mathrm{NS}$ & \multicolumn{3}{|c|}{$\mathrm{p}$-value $=0,130 \mathrm{NS}$} & & \multicolumn{5}{|c|}{ Fisher $(\mathrm{p}$-value $)=0,659 \mathrm{NS}$} \\
\hline
\end{tabular}

PPS: Potentiel Physique Supraliminaire; PPI: Potentiel Physique Infraliminaire; PPL:

Potentiel Physique Liminaire; n: Effectif; VO: Valeur Observée; VN: Valeur Normative, IMC: Indice de Masse Corporelle; IMG: Indice de Masse Grasse; \%: pourcentage; NS: Non Significative; S: Significative; kg: kilogramme; m: mètre; F: Fille; G: Garçon.

Des résultats statistiques analogues sont observés lorsqu'on considère le potentiel physique supraliminaire des jeunes dont l'âge oscille entre 22 à 25 ans. Ainsi, les garçons (IMC $=27,0 \mathrm{~kg} / \mathrm{m}^{2}, \mathrm{IMG}=22,34 \%$ de graisse) présentent une fréquence d'auto-acceptation corporelle basse, équivalente à celle de leurs homologues filles $\left(\mathrm{IMC}=30,83 \mathrm{~kg} / \mathrm{m}^{2}, \mathrm{IMG}=34,82 \%\right.$ de graisse) $(100 \%$ contre $83,3 \%$; $p$-value $=0,659$ ) (confère le Tableau VI).

Le sens des résultats sus-évoqués ne change pas, lorsque l'on examine les jeunes garçons et filles de potentiel physique liminaire. En d'autres termes, les garçons de potentiel physique liminaire (IMC $=21,43 \mathrm{~kg} / \mathrm{m}^{2}, \mathrm{IMG}=15,54 \%$ de graisse) acceptent leur corps autant que leurs homologues filles de potentiel physique identique (IMC $=22,04 \mathrm{~kg} / \mathrm{m}^{2}, \mathrm{IMG}=27,07 \%$ de graisse) $(63,6 \%$ contre 47,8\%; p-value=0,213) (confère le Tableau VI).

En somme, se référant aux résultats obtenus de façon générale, l'autoappréciation corporelle chez les individus jeunes dépendrait du niveau de leur IMC, IMG. En revanche, de manière spécifique, en considérant le sexe, l'autoappréciation corporelle fonctionnerait indépendamment des niveaux du potentiel physique des jeunes. Quel sens pourrait-on donner à ces résultats?

\section{Discussion}

L'étude a examiné l'auto-appréciation corporelle en relation avec le potentiel physique chez des jeunes âgés de 18 à 25 ans. Les résultats des observations relatives à cette susceptible relation sont particuliers. Les jeunes de 18 à 21 ans de potentiel physique liminaire présentent une auto- 
appréciation corporelle qui diffère de celle de leurs pairs de potentiel physique infraliminaire et supraliminaire ( $\mathrm{p}$-value < 0,05) (confère le Tableau I). L'on observe également une coïncidence entre ceux de 22 à 25 ans de potentiel physique variable ( $\mathrm{p}$-value $<0,05$ ) (confère le Tableau II). Les résultats des comparaisons suivant le sexe ne révèlent pas de différence statistiquement significative entre les jeunes garçons et les jeunes filles, quel que soit le potentiel physique (liminaire, supraliminaire ou infraliminaire) et les tranches d'âge (18-21 ou 22-25 ans). (p-value > 0,05, confère les Tableaux III, IV, V et VI).

La différence d'auto-appréciation corporelle, selon les niveaux du potentiel physique observée entre les jeunes de façon générale, semble tirer son explication dans divers facteurs. L'un d'entre ceux-ci est relatif au degré de conformité entre la structure du corps de l'individu et le modèle de corps établi par la société. Dans cette perspective, selon Pajor et al. (2015), les individus dont leur corps est identique au modèle prescrit par la société ont tendance à avoir une confiance, une assurance en leur corps et, par ricochet, ont un jugement élevé de leur soi corporel, comparativement à ceux dont le corps est en deçà ou au-dessus des valeurs du modèle de corps.

La non coïncidence observée, en générale, chez les jeunes de potentiel physique différent, dans l'évaluation de leur corps, pourrait être attribuable au choix par ces jeunes d'un modèle distinct de corps inné. Dans une même orientation, les différences observées semblent tirer leurs sources dans l'effet de l'âge du développement physique. Ainsi, les analyses de Gupta (2011) révèlent que les jeunes post-adolescents ont tendance à s'imposer. De ce fait, ceux-ci entreprennent des actions les amenant à ressembler à un modèle, afin de s'accepter physiquement. Tous ceux-ci s'avèrent être des éléments explicatifs de la variation de l'auto-acceptation corporelle suivant le niveau d'IMC et d'IMG.

Les résultats des analyses de la fréquence de l'auto-appréciation corporelle observés dans la présente étude seraient loin de s'écarter de ceux évoqués par Cash et Pruzinsky (2002). Ces auteurs révèlent dans leur examen que, les individus qui rejettent leur corps sont ceux qui présentent un corps déstructuré. Par contre, ceux de corps équivalent au seuil acceptent le plus leur corps. Ces observations sont identiques à celles réalisées par Muda et al. (2015), Lipowska et al. (2015). Selon ces auteurs, les sujets qui ont un potentiel corporel adéquat (IMC et IMG normaux) présentent une plus forte satisfaction corporelle que leurs pairs ayant un potentiel corporel inapproprié (IMC et IMG faibles ou élevés).

Autrement dit, le jugement que le jeune porte sur son corps dépendrait des acquisitions corporelles réalisées, c'est-à-dire le potentiel physique. De ce fait, les jeunes ayant construit un rapport négatif à leur corps seraient plus enclins à une auto-appréciation corporelle négative. Ces conclusions se 
répètent dans les recherches réalisées par Lionel \& Michel. (2015) en France, de Seidah et al. (2004) au Canada et de Małkowska-Szkutnik et al. (2012) en Pologne. Les résultats de ces observations confortent l'information selon laquelle les jeunes de densité physique conforme à la norme acceptent leur profil corporel que leurs homologues de densité physique en deçà de celui pris comme référence biophysique ou sociocorporelle.

Le sens des résultats change lorsque l'on fait intervenir le sexe. En effet, La superposition des jeunes de niveaux de potentiel physique différents concernant l'acceptation de leur profil corporel pourrait être due à diverses variables sociobiologiques. L'un des facteurs susceptible d'expliquer l'indifférence entre les deux groupes (garçons et filles) est celui évoqué par Gondoli et al. (2011), en l'occurrence la similarité du stade de développement biomorphologique. Selon ces auteurs, les individus, présentant une période de croissance et de maturation identique, ont tendance à définir leur corps par rapport à un profil corporel acquis, commun à eux. Par conséquent, ils s'influencent réciproquement, quant à l'acceptation ou non de leur profil physique. L'équivalence de l'âge biochronologique est celle qui apporte également un sens à l'égalité entre les jeunes garçons et leurs homologues filles de potentiel physique similaire, à propos de l'appréciation corporelle. Selon Grogan (2008), les jeunes filles et leurs pairs garçons à cet âge (18 à 25 ans) se caractérisent par une propension à recourir aux mêmes techniques ou activités de développement de leur corps. Le choix ou l'utilisation par les deux groupes de jeunes de procédés générationnels communs à leur âge pour donner une orientation à leur corps entrainerait un auto-jugement corporel qui est loin de varier d'un garçon à l'autre ou d'une fille à une autre.

La concordance des jugements que les jeunes filles et leurs homologues garçons portent sur leur corps, en dépit des écarts soupçonnés entre les potentiels physiques serait provoquée par la superposition des temps de vie. Dans cette perspective, le dimorphisme sexuel est désigné comme étant une variable exerçant un effet différent sur 1'auto-jugement corporel (Jackson, 2002). Cet auteur indique, dans son travail, que la fille, contrairement au garçon, se caractériserait par une acceptation moins régulière de son corps. Ce rejet corporel plus fréquent chez la fille est sous-tendu, selon ce chercheur, par un regard minutieux, millimétré que cette dernière porte sur la structuration de son corps. Ainsi, un gain de masse corporelle ou grasse, si petite soit-elle, est susceptible de créer chez la jeune fille une gêne profonde envers sa silhouette et, par ricochet, une auto-évaluation corporelle négative.

En somme, les réponses à la question relative à l'impact supposé du potentiel physique sur l'auto-évaluation corporelle se révèlent, considérant les résultats des travaux antérieurs et ceux obtenus dans le présent travail, divergentes. Les mécanismes mis en évidence dans l'explication des résultats de l'étude ne seraient forcément extrapolables. 


\section{Conclusion}

La question examinée dans la présente étude transversale et analytique est relative à l'auto-appréciation que les jeunes soumettent à leur corps en fonction de leur capacité physique adaptative. Les données collectées auprès de 181 jeunes garçons et filles âgés de 18 à 25 ans ont été traitées à l'aide de méthodes standardisées.

Les résultats obtenus dans cette étude montrent que d'emblée, les jeunes de potentiel physique liminaire acceptent leur corps que leurs pairs de potentiel physique supraliminaire et infraliminaire. L'issue des comparaisons change, lorsqu'on considère le sexe. Dans cette perspective, l'autoappréciation corporelle des jeunes garçons de potentiel physique liminaire, supraliminaire ou infraliminaire ne diffère pas de celle des jeunes filles de potentiel physique analogue.

La densité physique influencerait considérablement l'autoappréciation corporelle lorsqu'on considère l'ensemble des jeunes. En revanche, lorsqu'on fait intervenir le sexe, l'auto-jugement corporel ne change pas quand on passe d'un niveau de densité physique à un autre. Le nombre moins important de sujets examinés dans le présent travail amène, toutefois, à une certaine prudence, concernant l'infirmation de l'hypothèse de recherche. Des études ultérieures, considérant un échantillon de sujets de taille significative, comprenant des sujets issus de milieux socio-économiques différents, apporteraient des réponses plus précises à la problématique explorée, c'est-à-dire l'auto-appréciation corporelle juvénile.

\section{Remerciements}

Les auteurs de l'étude remercient la direction de l'Institut des Sciences Anthropologique de Développement (ISAD) de l'Université Félix HouphouëtBoigny qui a accepté que la collecte des données relatives à la question examinée se réalise au sein dudit institut. Ils sont également reconnaissants à l'égard des étudiants de l'ISAD qui ont contribué volontairement à l'échantillon de sujets sur lequel a porté la recherche.

\section{References:}

1. Bradaric, C., Kuhs, K., Groha, P., Dommasch, M., Langwieser, N., Haller, B.,...Ibrahim, T. (2015). Endovascular therapy for stenoocclusive subclavian and innominate artery disease. Circulation Journal, 79 (3), 537-543. doi: 10.1253/circj.CJ-14-0855.

2. Cash, F. T., \& Pruzinsky, T. (2002). Body image: a handbook of theory research and Clinical Practice. New York: The Guilford press.

3. Derraik, J. G., Lundgren, M., Cutfield, W. S., \& Ahlsson, F. (2016). Body Mass Index, Overweight, and Obesity in Swedish Women Born 
Post-term. Paediatric and Perinatal Epidemiology, 30 (4), 320-324. doi: 10.1111/ppe.12292.

4. Gondoli, D. M., Corning, A. F., Blodgett, S., Elizabeth. H., Bucchianeri, M. M., \& Fitzsimmons, E. E. (2011). Heterosocial Involvement, Peer Pressure for Thinness, and Body Dissatisfaction among Young Adolescent Girls. Body Image, 8(2), 143-148. doi: 10.1016/j.bodyim.2010.12.005.

5. Grogan, S. (2008). Body image: Understanding body dissatisfaction in men, women and children. New York: Routledge.

6. Gupta, C. (2011). The Relation between Body Image Satisfaction and Self-esteem to Academic Behaviour in Adolescents and Preadolescents. Manitoba: University of Manitoba.

7. Institut National de la Statistique (INS) (2014). Recensement générale de la population et de l'habitat en Côte d'Ivoire. Abidjan: INS.

8. Jackson, L. A. (2002). Physical attractiveness: a sociocultural perspective. In T. Cash, \& T. Pruzinsky (Eds.), Body image: a handbook of theory, research, and clinical practice (pp. 13-21). New York: The Guilford press.

9. Jáuregui, L. I., \& Bolaños, R. P. (2011). Body image and quality of life in a Spanish population. International Journal of General Medicine, 25 (4), 63-72. doi: 10.2147/IJGM.S16201.

10. Karastergiou. K., \& Pramyothin. P. (2016). What can we learn from Interventions that change fat distribution? Current Obesity Reports, 5(2), 271-81. doi: 10.1007/s13679-016-0215-x.

11. Lentillon-Kaestner, V., Berchtold, A., Rousseau, A., \& Ferrand, C. (2010). Validity and reliability of the french shortened versions of the body shape questionnaire (version française). Journal of Personality Assessment, 96 (4), 471-477.

12. Lionel, D., \& Michel, M. (2015). Image corporelle et estime de soi: étude auprès de lycéens français. Bulletin de Psychologie, 5 (509), 321-334. DOI:10.3917/bupsy.509.0321.

13. Lipowska, M., \& Lipowski, M. (2015). Narcissism as a moderator of satisfaction with body image in young women with extreme underweight and obesity. PloS One, 10 (5): e0126724. doi:10.1371/ journal.pone.0126724.

14. Małkowska-Szkutnik, A., Gajewski, J., Mazur, J. \& Gajewska, K. (2012). Self-esteem predictors in adolescents with diabètes. Medycyna Wieku Rozwojowego, 16(1), 35-46.

15. Mercier, M., \& Schraub, S. (2005). Qualité de vie: quel outil de mesure? Vingt septième (27) ème journée de la Société française de Sénologie et de pathologie mammaire, 418-423. 
16. Monique, A. (2000). La variabilité de la taille et du poids chez l'homme: Moyenne statistique, normalité, normativité. Astern (30), 143-168.

17. Muda, W. A., Kuate, D., Jalil, R. A., Nik, W. S., \& Awang, S. A. (2015). Self-perception and quality of life among overweight and obese rural housewives in Kelantan, Malaysia. Health Quality of Life Outcomes, 13 (19). doi: 10.1186/s12955-015-0210-z.

18. Neumark-Sztainer, D., Paxton, J. S., Hannan, P. J., Haines, J., \& Story, M. (2006). Does body satisfaction matter? Five-year longitudinal associations between body satisfaction and health behaviors in adolescent females and males. Journal of Adolescent Health, Elsevier, 39 (2), 244-251.

19. Pajor, A. J., Broniarczyk-Dyła, G., \& Świtalska, J. (2015). Satisfaction with life, self-esteem and evaluation of mental health in people with tattoos or piercings. Psychiatria Polska, 49(3), 559-573. doi: 10.12740/PP/27266.

20. Pearl, R. L., White, M. A., \& Grilo, C. M. (2014). Overvaluation of shape and weight as a mediator between self-esteem and weight bias internalization among patients with binge eating disorder. Eating Behaviors, 15 (2), 259-261. doi: 10.1016/j.eatbeh.2014.03.005.

21. Pica, L. A., Traoré, I., Camirand, H., Laprise, P., Bernèche, F., Berthelot, M., \& Plante, N. (Eds) (2012). Enquête québécoise sur la santé des jeunes du secondaire 2010-2011. Le visage des jeunes d'aujourd'hui: leur santé physique et leurs habitudes de vie (Tome 1). Québec: Institut de la statistique du Québec.

22. Rasmussen, V. G., Kanstrup, H. L., \& Nielsen-Kudsk, J. E. (2015). Percutaneous balloon valvuloplasty in carcinoid pulmonary valve stenosis: Catheterization and Cardiovascular Interventions. Official journal of the Society for Cardiac Angiography and Interventions, 88 (7), 1174-1176. doi: 10.1002/ccd.26321.

23. Seidah, A., Bouffard, T., \& Vezeau, C. (2004). Perception de soi à l'adolescence: différences entre filles et garçons. Enfance, 56 (4), 405420. DOI:10.3917/enf.564.0405.

24. Service d'inscription de la scolarité centrale de l'Université Félix Houphouët-Boigny (UFHB) (2015-2016). Effectif des étudiants. Abidjan: UFHB.

25. Wang, X., \& Davidson, N. (2010). The upper and lower bounds of the prediction accuracies of ensemble methods for binary classification. Procedings of International Conference on Machine Learning and Application, 373-378. doi:10.1109/ICMLA.2010.62. 